\title{
Efeitos da música em pacientes com câncer em tratamento quimioterápico
}

\author{
Effects of music on patients with cancer under chemotherapy treatment
}

Efectos de la música en pacientes con cancer en tratamiento quimioterápico

Igor Luis Silva Miranda ${ }^{1 *}$, Filipe Dalboni Polito da Silva, Geovani Santos da Silva ${ }^{1}$, Léo Pedro Rufino ${ }^{1}$, Lucas Morais Batista ${ }^{1}$, Edilaine Assunção Caetano de Loyola ${ }^{1}$.

\section{RESUMO}

Objetivo: Identificar as evidências disponíveis na literatura científica sobre os efeitos da música em pacientes com câncer em tratamento quimioterápico. Métodos: Revisão integrativa da literatura, a partir do referencial teórico da Prática Baseada em Evidências, utilizando a questão norteadora: qual a temática das publicações sobre os efeitos da música em pacientes com câncer em tratamento quimioterápico? As bases acessadas foram: Biblioteca Virtual em Saúde (BVS), Biblioteca Nacional de Medicina dos Estados Unidos (PUBMED) e Biblioteca Cochrane (COCHRANE), com os descritores controlados "música", "musicoterapia", "neoplasias", "tratamento farmacológico", "ansiedade". Resultados: Foram identificados 142 artigos no período de 2015 a 2020. Após a leitura dos títulos e resumos foram excluídos, na primeira etapa, artigos que não se enquadraram ao tema e, na segunda etapa, após leitura dos artigos na íntegra e aplicando-se os critérios de seleção, se excluíram outras publicações por não se relacionarem ao objetivo proposto. Assim, 15 artigos representaram a amostra do estudo. Esses artigos centraram-se na perspectiva da redução dos efeitos ocasionados pelo tratamento quimioterápico, promovendo melhora da saúde física e mental desses pacientes. Considerações finais: A música atua na amenização das alterações comportamentais dos pacientes decorrentes da doença durante o tratamento, garantindo aos mesmos, melhores perspectivas no tratamento oncológico.

Palavras-chave: Música, Musicoterapia, Neoplasias, Tratamento farmacológico, Ansiedade.

\begin{abstract}
Objective: Identify the available evidences on literature about the effects of music in patients with cancer who are in chemotherapy treatment. Methods: Integrative literature review, based on theoretical framework of Evidence-Based Practice, using as guiding question: what is the theme of the publications about the music effects in patients who are suffering from chemotherapy treatment? The databases accessed was: Virtual Health Library (BVS), Medicine National Institutes of Health (PUBMED) and Cochrane Library (COCHRANE) with the controlled descriptors "music", "neoplasm", "drug treatment", "music therapy", "anxiety". Results: Have been identified 142 articles in the period between 2015 and 2020. After reading the titles and abstracts, in the first step, excluding articles that did not framed to the theme and, in the second step, after reading the full articles and applying the selection criteria, other publications were excluded because they were not related to the proposed study objective. Thus, 15 articles represented the study's sample. Those articles were centred in the perspective of reduction of the effects caused by chemotherapy treatment, promoting a better physical and mental health of these patients. Conclusion: The music acts softening the behavioral changes suffered by the patients due to treatment, ensuring to these individuals better perspectives in the oncology treatment.
\end{abstract}

Key words: Music, Neoplasm, Drug treatment, Music therapy, Anxiety.

1 Universidade José do Rosário Vellano (UNIFENAS), Alfenas - MG. *E-mail: igorluis12@outlook.com Programa Institucional de Bolsas (PIBIC/CNPq) da Universidade José do Rosário Vellano - UNIFENAS. 


\section{RESUMEN}

Objetivo: Identificar la evidencia disponible en la literatura científica sobre los efectos de la música en pacientes oncológicos sometidos a quimioterapia. Métodos: Revisión integrativa de la literatura, basada en el marco teórico de la Práctica basada en la evidencia, utilizando la pregunta orientadora: ¿cuál es el tema de las publicaciones sobre los efectos de la música en pacientes con cáncer sometidos a quimioterapia? Las bases de datos a las que se tuvo acceso fueron: Biblioteca Virtual de Salud (BVS), Biblioteca Nacional de Medicina de los Estados Unidos (PUBMED) y Biblioteca Cochrane (COCHRANE) con los descriptores controlados "música", "musicoterapia", "neoplasias", "tratamiento farmacológico", "ansiedad". Resultados: Han sido identificados 142 artículos en el período de 2015 a 2020. Luego de leer los títulos y resúmenes, los artículos que no encajaban en la temática fueron excluidos en la primera etapa y, en la segunda etapa, luego de la lectura completa de los artículos. y aplicando los criterios de selección, se excluyeron otras publicaciones por no estar relacionadas con el objetivo propuesto del estudio. Así, 15 artículos representaron la muestra de estudio. Estos artículos se centraron en la perspectiva de reducir los efectos provocados por la quimioterapia, promoviendo una mejora en la salud física y mental de estos pacientes. Consideraciones finales: La música actúa para paliar los cambios de comportamiento de los pacientes como consecuencia de la enfermedad durante el tratamiento, garantizándoles mejores perspectivas en el tratamiento del cáncer.

Palabras Clave: Música, Musicoterapia, Neoplasias, Tratamiento farmacológico, Ansiedad.

\section{INTRODUÇÃO}

O câncer é uma doença causada pela proliferação descontrolada de células de um órgão ou tecido. Esse processo gera componentes anormais que se multiplicam, formando um tumor, o qual pode desencadear diversos estágios de manifestação no organismo (RAMOS BF e LUSTOSA MA, 2009). Como existem vários tipos de câncer, essa patologia implica alterações de características individuais para cada paciente, as quais podem advir de fatores genéticos ou ambientais, sendo o último responsável por mais de $80 \%$ dos casos (BRASIL, 2020).

Nessa circunstância, a sociedade encara o diagnóstico de câncer como uma sentença de morte, ainda que, atualmente, a medicina já se mostra bastante eficaz para conduzir bons prognósticos com a descoberta precoce. Durante o tratamento, o paciente encara sensações de ansiedade, medo, insegurança e angústia, podendo ser identificados também após o término das intervenções (MERTZ BG, et al., 2012).

Deste modo, existem opções para a redução da ansiedade, como as Práticas Integrativas e Complementares (PICS), com destaque para a musicoterapia. As PICS são tratamentos que utilizam recursos terapêuticos baseados em conhecimentos tradicionais, direcionados à prevenção de várias doenças, sendo utilizadas, também, como tratamentos alternativos e complementares em algumas doenças crônicas, como o câncer. Assim, elas atuam na amenização dessas características, garantindo a esses pacientes melhores condições e perspectivas no tratamento oncológico (BRASIL, 2020).

De acordo com Benenzon R (1998), a musicoterapia objetiva estudar o indivíduo em sua integralidade, sendo o campo da medicina que utiliza o movimento, o som e a música, com o intuito de abrir canais de comunicação a fim de produzir efeitos terapêuticos, psicoprofiláticos e de reabilitação no ser humano.

Pouco se sabe sobre a intervenção da musicoterapia para atuar nos níveis de mediadores relacionados ao estresse, como o cortisol, considerando os estadiamentos da doença, em que os processos fisiopatológicos desencadeiam respostas orgânicas relacionadas a este hormônio que, quando sofre descontrole, gera mecanismos biológicos que atuam negativamente no organismo (GUYTON AC e HALL JE, 2017).

Diante dos aspectos físicos e emocionais, a interação musical é um recurso comunicacional que coopera com a diminuição de distúrbios psíquicos, emoções negativas e estilos de comportamento, beneficiando o âmbito terapêutico ao promover a mobilização de ondas sonoras, em frequência e amplitudes adequadas, que vão estimular diversos aparelhos orgânicos, agindo principalmente nos sistemas nervoso, endócrino e cardiovascular (BENENZON R, 1998). 
Nesse contexto, o desenvolvimento desta pesquisa se mostra essencial à medida que visa promover a avaliação de como a música pode interferir no tratamento de neoplasias, tornando-se relevante pela possibilidade de contribuir para o desenvolvimento de PICS que possam auxiliar os pacientes oncológicos, física e psicologicamente.

Essas considerações justificam o interesse em desenvolver uma revisão integrativa sobre a produção científica da temática acerca dos efeitos da música em pacientes com câncer em tratamento quimioterápico, na literatura científica, realizando um levantamento do conhecimento produzido na área para subsidiar a prática clínica do médico, bem como, levantar as necessidades de pesquisa e auxiliar no desenvolvimento de futuras investigações.

O aumento da complexidade e quantidade de informações na área da saúde, associada a necessidade de se basear cientificamente a prática clínica neste campo e definir critérios metodológicos mais precisos, que reúnam os conhecimentos apresentados em diversos estudos, levou a se buscar na literatura um referencial teórico que pudesse nortear o presente estudo. A Prática Baseada em Evidências (PBE), cujos princípios se vinculam ao trabalho de Archie Cochrane, é uma abordagem que incorpora os resultados oriundos de pesquisas, a competência clínica do profissional e as preferências do paciente para a tomada da decisão sobre a assistência à saúde. Relata sobre a busca, avaliação e aplicação de evidências científicas para o tratamento e gerenciamento do estado normal de saúde. O cuidado é guiado por meio de resultados de pesquisas e/ou consenso de especialistas (SILVEIRA RCCP e GALVÃO CM, 2005).

A PBE incentiva o profissional de saúde a buscar conhecimento científico, além de possibilitar a melhor qualidade na assistência prestada ao paciente, por meio do desenvolvimento de pesquisas ou aplicação na sua prática dos resultados encontrados na literatura (URSI ES e GALVÃO CM, 2006; GALVÃO CM, et al., 2002). A pesquisa médica na área oncológica, na assistência a pacientes que tiveram diagnóstico de câncer, é essencial para gerar a base de conhecimento que fundamenta a prática clínica, além de poder identificar o impacto da doença e do tratamento na vida das pacientes e familiares.

Frente a essas colocações o objetivo desse estudo foi identificar as evidências disponíveis na literatura científica, sobre os efeitos da música em pacientes com câncer em tratamento quimioterápico.

\section{MÉTODOS}

Para o alcance dos objetivos propostos optou-se pelo método da revisão integrativa de literatura, que se constitui em um instrumento da PBE. Por possibilitar a inclusão de artigos de métodos diversos, abrangendo estudos experimentais e não-experimentais para compreender por completo o fenômeno estudado, este tipo de revisão vem contribuindo substancialmente para a PBE na medicina (SOUZA MT, et al., 2010).

A revisão integrativa da literatura possibilita sumarizar as pesquisas encerradas e obter conclusões a partir de um tema de interesse. Uma revisão integrativa bem realizada exige os mesmos padrões de rigor, clareza e replicação utilizada nos estudos primários (BEYEA SC e NICOLL ELH, 1998). Na operacionalização dessa revisão, utilizamos as seguintes etapas: seleção das questões temáticas; estabelecimentos dos critérios para a seleção da amostra; representação das características da pesquisa original e organização dos dados; análise dos dados; interpretação dos resultados; apresentação da revisão (MENDES KDS, et al., 2008).

Diante do apresentado, formulamos a seguinte questão norteadora da pesquisa: "Qual a temática das publicações sobre os efeitos da música em pacientes com câncer em tratamento quimioterápico?"

Para a realização de uma pesquisa bibliográfica de qualidade, o primeiro passo é localizar a terminologia autorizada e reconhecida mundialmente. $O$ descritor controlado é parte de um vocabulário estruturado e organizado para facilitar o acesso à informação (PELLIZZON RF, 2004).

Assim, nesta pesquisa, inicialmente foi feita uma consulta aos Descritores em Ciências da Saúde (DeCS) "por descritor exato" para aquisição dos descritores universais e posterior utilização nas bases de dados, sendo que foram respeitados rigorosamente os artigos que apareceram após a utilização dos descritores de forma combinada "música" or "musicoterapia" and "neoplasias"; "música" or "musicoterapia" and "tratamento farmacológico"; "música" or "musicoterapia" and "ansiedade". 
Após esta primeira etapa, foi realizada uma nova busca nas bases de dados que integravam os artigos selecionados para verificação, via formulário iAH, utilizando palavras-chave e operadores boleanos, da mesma forma combinada, a saber: "intervenção musical" and "câncer"; "intervenção musical" and "quimioterapia"; "intervenção musical" and "ansiedade social".

O levantamento bibliográfico foi realizado pela internet nas bases de dados da BVS, PUBMED e COCHRANE; no campo "onde", optou-se por "todas as fontes".

Os critérios para a seleção da amostra foram: artigos publicados em língua portuguesa; língua inglesa, artigos que abordem a temática dos efeitos da música em pacientes com câncer em tratamento quimioterápico; periódicos indexados nas bases de dados que compõem a BVS; artigos na íntegra publicados no período de 2015 a 2020; todo artigo independente do método de pesquisa utilizado.

O levantamento bibliográfico nas bases de dados ocorreu no mês de outubro de 2020. A coleta de dados foi realizada em duas etapas. A primeira se baseou na busca avançada nas bases de dados, sendo identificados 142 artigos, para o período de 2015 a 2020 seguindo os critérios de inclusão e exclusão. Nesta primeira etapa, realizou-se a leitura dos títulos e resumos, resultando na exclusão de 96 artigos por não se enquadrarem ao tema, totalizando 46 artigos elegíveis.

Na segunda etapa, procedeu-se a leitura dos artigos na íntegra e aplicando-se os critérios de seleção, se excluíram outras 31 publicações por não se relacionarem ao objetivo proposto do presente estudo. Os estudos duplicados nas bases de dados foram considerados uma única vez. Assim, 15 artigos foram selecionados, representando a amostra do estudo (Tabela 1).

Tabela 1 - Distribuição dos artigos obtidos nas bases de dados por refinamento.

\begin{tabular}{cccccc}
\hline $\begin{array}{c}\text { BASES DE } \\
\text { DADOS }\end{array}$ & $\begin{array}{c}\text { ARTIGOS } \\
\text { LOCALIZADOS }\end{array}$ & $\begin{array}{c}\text { ARTIGOS } \\
\text { EXCLUÍDOS NA } \\
\text { PRIMEIRA ETAPA }\end{array}$ & $\begin{array}{c}\text { ARTIGOS } \\
\text { ELEGÍVEIS }\end{array}$ & $\begin{array}{c}\text { ARTIGOS } \\
\text { EXCLUÍDOS NA } \\
\text { SEGUNDA } \\
\text { ETAPA }\end{array}$ & $\begin{array}{c}\text { ARTIGOS } \\
\text { SELECIONADOS }\end{array}$ \\
\hline BVS & 44 & 31 & 13 & 7 & 6 \\
PUBMED & 30 & 24 & 6 & 3 & 3 \\
COCHRANE & 68 & 41 & 27 & 21 & 6 \\
\hline TOTAL & 142 & 96 & 46 & 31 & 15 \\
\hline
\end{tabular}

Fonte: Miranda ILS, et al., 2020.

Após a releitura de cada um dos artigos selecionados, foi realizada a caracterização, compilação e fichamento dos mesmos, por meio do preenchimento de um instrumento validado contendo, dentre outras variáveis: título do artigo, identificação do periódico, ano de publicação, idioma, país onde o estudo foi realizado, metodologia empregada, nível de evidência, bem como seus resultados e recomendações para a prática (URSI ES, 2005). Para reduzir possíveis erros de interpretação dos resultados, os cinco pesquisadores realizaram a leitura e preenchimento do instrumento de forma individual, os quais foram posteriormente comparados, não havendo divergências.

Para a avaliação dos níveis de evidência foi utilizada a classificação de Melnyk BM e Fineout-Overholt E (2018), sendo o nível 1 correspondente às revisões sistemáticas ou metanálises de ensaios clínicos relevantes; o nível 2 trata-se de evidências oriundas de pelo menos um ensaio clínico randomizado controlado bem delineado; o nível 3 confere ensaios clínicos bem delineados sem randomização; o nível 4 diz respeito aos estudos de coorte e de caso-controle bem delineados; o nível 5 às revisões sistemáticas de estudos descritivos e qualitativos; o nível 6 refere às evidências derivadas de um único estudo qualitativo ou descritivo e, por fim, o nível 7 corresponde a relatório de comitês de especialistas ou opinião de autoridades.

Para a apresentação dos resultados e síntese dos artigos que atenderam aos critérios de inclusão, foi utilizado um quadro sintetizado especialmente construído para este fim, composto pelos seguintes aspectos: título, o autor, ano de publicação, objetivo do estudo, resultados e conclusões. A discussão dos dados obtidos 
foi feita de forma descritiva, favorecendo ao leitor a avaliação da aplicabilidade da revisão integrativa formulada, de forma a atingir o objetivo desse método, ou seja, impactar positivamente na qualidade da atuação da medicina, fornecendo subsídios ao médico na sua prática cotidiana.

\section{RESULTADOS E DISCUSSÃO}

Inicialmente realizou-se uma caracterização dos artigos selecionados e identificação dos enfoques temáticos utilizados nos mesmos.

Dentre os estudos selecionados, sete $(46,66 \%)$ são ensaios clínicos randomizados controlados, quatro (26,66\%) são revisões sistemáticas, dois (13,66\%) estudo quase experimental, um (6,66\%) estudo piloto para ensaio clínico randomizado, um (6,66\%) estudo prospectivo. Com relação à autoria, totalizaram 94 autores dos trabalhos.

Constatou-se que todos os artigos selecionados são trabalhos originais, o que mostra a importância do presente estudo em demonstrar uma revisão integrativa para sintetizar o conhecimento adquirido na área.

Com relação à formação, trinta $(46,15 \%)$ são médicos, onze $(16,92 \%)$ enfermeiros, cinco $(7,99 \%)$ fisioterapeutas, cinco $(7,69 \%)$ músicos, quatro $(6,15 \%)$ psicólogos, três $(4,61 \%)$ musicoterapeutas, três $(4,61 \%)$ bioestatísticos, um (1,63\%) epidemiologista e um (1,63\%) administrador, um (1,63\%) acadêmico de medicina, um $(1,63 \%)$ matemático.

Como característica importante, a maioria dos pesquisadores são da área médica e de enfermagem, o que revela a importância e necessidade de buscarem-se recursos de pesquisas voltadas para o tema.

No que se refere à titulação, quarenta e oito $(73,84 \%)$ são doutores, doze $(18,40 \%)$ mestres, cinco $(7,69 \%)$ especialistas. Vale ressaltar a titulação dos autores, sendo a maioria doutores e mestres atuantes na docência, evidenciando o exercício universitário na complementação de falhas existentes na atenção integral voltada para os pacientes com câncer.

Quanto ao ano de publicação dos artigos, verifica-se que foram publicados no período de 2015 a 2020 , sendo um $(6,66 \%)$ publicado em 2015 , dois $(13,33 \%)$ em 2016 , quatro $(26,66 \%)$ em 2017 , dois $(13,33 \%)$ em 2018, quatro (26,66\%) em 2019 e dois (13,33\%) em 2020.

Constatou-se que os estudos eram compostos de equipes multidisciplinares, as quais desempenharam papel relevante na condução dos manejos realizados para avaliar a intervenção da música no tratamento oncológico. Todos os estudos selecionados foram realizados entre os anos de 2015 e 2020, sendo estes pertinentes ao foco de evidenciar os efeitos benéficos da musicoterapia na atualidade.

De acordo com o referencial utilizado para a avaliação do nível de evidência Melnyk BM e Fineout-Overholt $E(2018)$, cinco (33,33\%) estudos apresentaram nível de evidência 1, correspondente às revisões sistemáticas ou metanálises de ensaios clínicos relevantes; oito $(53,33 \%)$ apresentaram nível de evidência 2, evidências oriundas de pelo menos um ensaio clínico randomizado controlado bem delineado; um (6,66\%) apresentou nível de evidência 3, ensaios clínicos bem delineados sem randomização; um (6,66\%) apresentou nível de evidência 4, estudos de coorte e de caso-controle bem delineados. Os bons níveis de vidência apresentados pela maioria dos estudos demonstram pesquisas com bom desenho metodológico e que têm resultados importantes para o avanço da implantação das PICS na área oncológica (Quadro 1). 


\begin{tabular}{|c|c|c|c|}
\hline TIPO DO ESTUDO & AUTORES/ PAÍS/ ANO & OBJETIVO DO ESTUDO & RESULTADOS E CONCLUSÕES \\
\hline $\begin{array}{l}\text { Estudo quase } \\
\text { experimental }\end{array}$ & $\begin{array}{c}\text { CHEN S, et al./ China/ } \\
2017\end{array}$ & $\begin{array}{l}\text { Determinar os efeitos da intervenção musical em grupo e } \\
\text { da intervenção musical auto-dirigida sobre a ansiedade, } \\
\text { depressão e avaliação cognitiva de mulheres com câncer } \\
\text { de mama durante a quimioterapia. }\end{array}$ & $\begin{array}{l}\text { A intervenção musical em grupo se mostrou um sistema } \\
\text { de suporte e amparo eficaz no alívio dos efeitos } \\
\text { quimioterápicos e angústia, melhorando os efeitos } \\
\text { cognitivos de pacientes com neoplasias mamárias. }\end{array}$ \\
\hline $\begin{array}{l}\text { Estudo quase } \\
\text { experimental }\end{array}$ & $\begin{array}{l}\text { TOCCAFONDI A, et al. } \\
\text { / Itália/ } 2017\end{array}$ & $\begin{array}{l}\text { Avaliar o efeito de uma única intervenção musical ao vivo } \\
\text { seguida de uma avaliação sobre a carga psicológica e } \\
\text { sobre o bem-estar de pacientes hospitalizados com } \\
\text { câncer. }\end{array}$ & $\begin{array}{l}\text { A intervenção se mostrou eficaz, com melhoras no bem- } \\
\text { estar dos pacientes hospitalizados com câncer, sendo } \\
\text { facilmente replicável e de baixo custo. }\end{array}$ \\
\hline $\begin{array}{l}\text { Estudo piloto para } \\
\text { ensaio clínico } \\
\text { randomizado }\end{array}$ & $\begin{array}{l}\text { ROBB S, et al./ EUA/ } \\
2016\end{array}$ & $\begin{array}{l}\text { Avaliar a viabilidade e aceitabilidade de uma intervenção } \\
\text { de engajamento musical ativo oferecida pelos pais para } \\
\text { crianças pequenas com câncer, examinando as } \\
\text { mudanças no sofrimento emocional das crianças, por } \\
\text { meio das expressões faciais, e dos pais, através do } \\
\text { humor e sintomas traumáticos do estresse. }\end{array}$ & $\begin{array}{l}\text { Foi possível realizar a intervenção pois os pais } \\
\text { ofereceram a intervenção de engajamento musical ativo, } \\
\text { mas, apesar do menor sofrimento emocional } \\
\text { apresentado pelas crianças, não houve benefícios para } \\
\text { os pais, que segundo os dados das entrevistas, não } \\
\text { aceitaram bem a intervenção. }\end{array}$ \\
\hline Revisão sistemática & $\begin{array}{l}\text { BARBOSA NERES C, } \\
\text { et al./ Brasil/ } 2019\end{array}$ & $\begin{array}{l}\text { Revisar sistematicamente os estudos e determinar a } \\
\text { efetividade da musicoterapia na redução da ansiedade } \\
\text { de pacientes oncológicos }\end{array}$ & $\begin{array}{l}\text { A musicoterapia é efetiva na redução da ansiedade de } \\
\text { pessoas com câncer. No entanto, mais estudos com } \\
\text { novas tecnologias e mais detalhes sobre a intervenção } \\
\text { são necessários para a confirmação dos resultados. }\end{array}$ \\
\hline Estudo prospectivo & $\begin{array}{l}\text { GENCER D, et } \\
\text { al./Alemanha/ } 2019\end{array}$ & $\begin{array}{l}\text { Avaliar os efeitos positivos de intervenção musical em } \\
\text { aspectos de qualidade de vida ou gerenciamento de } \\
\text { sintomas em pacientes oncológicos em quimioterapia. }\end{array}$ & $\begin{array}{l}\text { Pacientes relataram interesse em intervenção musical } \\
\text { adicional durante o tratamento do câncer, devido à } \\
\text { redução potencial da sua carga de sintomas. }\end{array}$ \\
\hline $\begin{array}{l}\text { Ensaio clínico } \\
\text { randomizado }\end{array}$ & $\begin{array}{l}\text { BURNS DS, et al./ } \\
\text { EUA/ } 2018\end{array}$ & $\begin{array}{l}\text { Examinar os efeitos da musicoterapia e das imagens de } \\
\text { suporte em comparação com a música preferida de } \\
\text { pacientes oncológicos em resposta à angústia, } \\
\text { ansiedade e depressão e busca de benefícios. }\end{array}$ & $\begin{array}{l}\text { A música e as imagens e as experiências preferidas de } \\
\text { escuta musical podem servir a diferentes propósitos } \\
\text { terapêuticos, acessar e ativar diferentes recursos do } \\
\text { paciente e podem ser úteis em diferentes estágios do } \\
\text { tratamento. }\end{array}$ \\
\hline $\begin{array}{l}\text { Ensaio clínico } \\
\text { randomizado }\end{array}$ & $\begin{array}{l}\text { LIU H, et al. / China/ } \\
\qquad 2019\end{array}$ & $\begin{array}{l}\text { Avaliar o efeito da redução do estresse baseada no } \\
\text { método Atenção Plena associada à Musicoterapia em } \\
\text { pacientes com osteosarcoma. }\end{array}$ & $\begin{array}{l}\text { O programa de intervenção aliviou de forma significativa } \\
\text { as complicações psicológicas e fisiológicas em pacientes } \\
\text { com osteossarcoma. Em específico, o estudo revelou } \\
\text { que, em } 8 \text { semanas do programa, houve redução da dor } \\
\text { e ansiedade, além do aprimoramento da qualidade do } \\
\text { sono nos pacientes. }\end{array}$ \\
\hline $\begin{array}{l}\text { Revisão sistemática } \\
\text { e metanálise }\end{array}$ & $\begin{array}{l}\text { WANG X, et al./ China/ } \\
2018\end{array}$ & $\begin{array}{l}\text { Avaliar os efeitos da intervenção musical na saúde } \\
\text { mental e física de pacientes com câncer de mama. }\end{array}$ & $\begin{array}{l}\text { A revisão demonstrou indícios de que a musicoterapia } \\
\text { promove aprimoramento na saúde mental e física de } \\
\text { pacientes com câncer de mama. }\end{array}$ \\
\hline
\end{tabular}




\begin{tabular}{|c|c|c|c|}
\hline TIPO DO ESTUDO & AUTORES/ PAÍS/ ANO & OBJETIVO DO ESTUDO & $\begin{array}{l}\text { RESULTADOS E CONCLUSÕES } \\
\end{array}$ \\
\hline $\begin{array}{l}\text { Ensaio clínico } \\
\text { randomizado } \\
\text { controlado }\end{array}$ & $\begin{array}{l}\text { BIELIGMEYER S, et } \\
\text { al./ Alemanha/ } 2018\end{array}$ & $\begin{array}{l}\text { Avaliar os efeitos imediatos de uma música ouvida no } \\
\text { leito, no que diz respeito ao bem-estar subjetivo, bem } \\
\text { como ao calor e dor no corpo. }\end{array}$ & $\begin{array}{l}\text { A intervenção no leito de som melhorou o bem-estar } \\
\text { momentâneo e causou a auto-percepção de alterações } \\
\text { fisiológicas associadas ao relaxamento além dos } \\
\text { benefícios do simples tempo de descanso (condição de } \\
\text { controle). }\end{array}$ \\
\hline Revisão sistemática & $\begin{array}{c}\text { GREENLEE H, et al / } \\
\text { EUA/ } 2017\end{array}$ & $\begin{array}{l}\text { Avaliar o uso de terapias integrativas para indicações } \\
\text { clínicas específicas durante e após o tratamento do } \\
\text { câncer de mama. }\end{array}$ & $\begin{array}{l}\text { Essas terapias podem ser incorporadas como uma } \\
\text { opção de atendimento aos pacientes, especialmente } \\
\text { quando há um controle ineficaz dos sintomas em } \\
\text { pacientes oncológicos. }\end{array}$ \\
\hline Revisão sistemática & $\begin{array}{l}\text { BRADT J, et al / EUA/ } \\
2016\end{array}$ & $\begin{array}{l}\text { Avaliar e comparar os efeitos das intervenções de } \\
\text { musicoterapia e resultados físicos e psicológicos em } \\
\text { pessoas com câncer. }\end{array}$ & $\begin{array}{l}\text { As intervenções musicais podem ter efeitos benéficos na } \\
\text { ansiedade, dor, fadiga e qualidade de vida em pessoas } \\
\text { com câncer. }\end{array}$ \\
\hline $\begin{array}{l}\text { Ensaio clínico } \\
\text { randomizado }\end{array}$ & $\begin{array}{l}\text { LIMA TU, et al / Brasil/ } \\
\qquad 2020\end{array}$ & $\begin{array}{l}\text { Determinar o efeito da intervenção musical sobre os } \\
\text { sintomas, eventos adversos e qualidade de vida em } \\
\text { pacientes com câncer de mama em tratamento } \\
\text { quimioterápico. }\end{array}$ & $\begin{array}{l}\text { Os pacientes do grupo intervenção apresentaram } \\
\text { melhores escores de qualidade de vida de escalas } \\
\text { funcionais, quando comparados ao grupo controle. } \\
\text { Depressão, vômitos e ansiedade foram menos } \\
\text { observados no grupo intervenção. Todos os participantes } \\
\text { do grupo intervenção relataram melhoras em parâmetros } \\
\text { subjetivos. }\end{array}$ \\
\hline $\begin{array}{l}\text { Ensaio clínico } \\
\text { randomizado } \\
\text { controlado }\end{array}$ & $\begin{array}{l}\text { BRO ML, et al / } \\
\text { Dinamarca/ } 2019\end{array}$ & $\begin{array}{l}\text { Examinar se ouvir música ao vivo ou pré-gravada diminui } \\
\text { a ansiedade durante a quimioterapia em pacientes com } \\
\text { linfoma recém-diagnosticados. }\end{array}$ & $\begin{array}{l}\text { Resultados sugerem que a música ao vivo preferida pelo } \\
\text { paciente reduz a ansiedade entre os pacientes com } \\
\text { linfomas malignos submetidos à quimioterapia. A } \\
\text { habilidade musical neste grupo de pacientes com câncer } \\
\text { parece não ser um fator determinante para o efeito da } \\
\text { intervenção musical. }\end{array}$ \\
\hline $\begin{array}{l}\text { Ensaio clínico } \\
\text { randomizado }\end{array}$ & $\begin{array}{l}\text { MASSIMILIANI V, et al/ } \\
\text { Alemanha/2017 }\end{array}$ & $\begin{array}{l}\text { Investigar os efeitos da intervenção musical sobre alguns } \\
\text { parâmetros fisiológicos e psicológicos, especialmente } \\
\text { ansiedade, em pacientes com câncer durante a } \\
\text { administração de quimioterapia. }\end{array}$ & $\begin{array}{l}\text { A intervenção musical durante a administração da } \\
\text { quimioterapia diminuiu o estado de ansiedade e } \\
\text { frequência cardíaca em pacientes oncológicos, } \\
\text { mostrando que uma simples intervenção ambiental pode } \\
\text { promover bem-estar psicológico e melhora na qualidade } \\
\text { de vida }\end{array}$ \\
\hline $\begin{array}{l}\text { Ensaio clínico } \\
\text { randomizado } \\
\text { controlado }\end{array}$ & $\begin{array}{l}\text { TANG, H-K, et al/ } \\
\text { China/ } 2015\end{array}$ & $\begin{array}{l}\text { Observar as terapias musicais em seis passos para a } \\
\text { mudança da ansiedade e qualidade do sono em } \\
\text { pacientes com câncer de pulmão sob tratamento } \\
\text { quimioterápico. }\end{array}$ & $\begin{array}{l}\text { A musicoterapia em seis passos pode reduzir } \\
\text { efetivamente a ansiedade, aliviar a dor após a } \\
\text { quimioterapia e melhorar a qualidade do sono em } \\
\text { pacientes com câncer sob tratamento quimioterápico. }\end{array}$ \\
\hline
\end{tabular}

Fonte: Miranda ILS, et al., 2020. 
Verificou-se que todos os artigos centraram-se em uma perspectiva principal no que concerne aos efeitos da música em pacientes com câncer em tratamento quimioterápico: a redução dos efeitos ocasionados pelo tratamento quimioterápico, como o estresse, a ansiedade, dor, fadiga e aumento do bem estar, promovendo dessa forma, uma melhora da saúde física e mental dessas pacientes. Os artigos mostraram resultados efetivamente benéficos a curto prazo de aplicação da prática integrativa por meio da música. Entretanto, os achados quanto a melhora dos sintomas apresentados pelos pacientes foi alcançada em curto tempo, demonstrando, assim, a necessidade de uma aplicação a longo prazo dessa terapia para confirmar o efeito e de novas pesquisas nessa área para complementação dos resultados e aplicação na prática clínica (BIELIGMEYER S, et al., 2018).

A probabilidade de desenvolvimento do câncer durante toda a vida de um indivíduo é de $44 \%$, no caso dos homens, e $38 \%$ no caso das mulheres. Bradt J, et al. (2016) verificaram na experiência dos pacientes diagnosticados e portadores de neoplasias a existência de influências físicas, como distúrbios de apetite, dificuldade de deglutição, náusea, vômitos, constipação, diarreia, dispneia, fadiga, insônia, fraqueza muscular e dormência. As influências psicológicas causadas pela doença ou pelo tratamento farmacológico, tais como o medo, a ansiedade e a angústia, foram apresentadas em 30 a 50\% dos portadores de patologias neoplásicas (BARBOSA NERES C, et al., 2020; GENCER D, et al., 2019; BURNS D, et al., 2018; TOCCAFONDI A, et al., 2018).

Os pacientes apresentaram comumente sofrimento e tendem a visualizar o câncer como uma doença que inevitavelmente levará à morte de quem a possui. Os estudos mostraram que o tabu existente ao redor desta doença, assim como o impacto psicossocial da mesma contribuem para o desenvolvimento de uma doença psíquica que influencia negativamente no tratamento, a depressão (LIU H, et al., 2019; CHEN S, et al., 2018).

A música foi apontada nos estudos como instrumento já conhecido e utilizado para a mudança de comportamentos, sentimentos ou fatores fisiológicos por décadas. No entanto, há uma diferença entre a musicoterapia e a medicina musical. A musicoterapia aparenta possuir maior eficácia no controle dos efeitos provocados pelo câncer e suas decorrências devido ao seu caráter mais individual, onde um aplicador devidamente treinado seleciona o método e o estilo musical mais apropriados para cada paciente. Essa técnica usa o processamento associativo da memória para aliviar emoções negativas através da ocupação dos centros de atenção cerebrais com estímulos relaxantes e significantes (BARBOSA NERES C, et al., 2020; CHEN S, et al., 2018).

A música revelou sua efetividade em várias etapas da doença, como durante a radioterapia, quimioterapia, cirurgia e transplante (BARBOSA NERES C, et al., 2020; TOCCAFONDI A, et al., 2018). Isso se deve à redução da ansiedade, depressão, raiva, dores físicas e fadiga, também sendo verificada pela melhora da qualidade de vida dos pacientes oncológicos (GREENLEE H, et al., 2017; MASSIMILIANI V, et al., 2017; BRADT J, et al., 2016; ROBB S, et al., 2016). A justificativa apontada para o uso da musicoterapia em conjunto com outros métodos terapêuticos foi a de que essa técnica, além de ser efetiva, também se mostrou segura e não invasiva para os pacientes em questão (LIMA TU, et al., 2020; LIU H, et al., 2019).

A música, as imagens e as experiências preferidas de escuta musical podem servir a diferentes propósitos terapêuticos, acessar e ativar diferentes recursos do paciente e podem ser úteis em diferentes estágios do tratamento (BURNS D, et al., 2018; TANG H-k, et al., 2015). O estudo cujo objetivo foi examinar se ouvir música ao vivo ou pré-gravada diminui a ansiedade durante a quimioterapia em pacientes com linfoma recémdiagnosticados, os autores encontraram que escutar a música ao vivo preferida pelos pacientes reduziu a ansiedade entre aqueles em quimioterapia; entretanto, a música pré-gravada, por sua vez, não obteve um resultado significativo em ralação aos pacientes sem a intervenção. É válido ressaltar, ainda, que a habilidade musical parece não ser um fator determinante para o efeito da intervenção em questão (BRO ML, et al., 2019).

O uso da música de forma passiva, no entanto, pode influenciar negativamente o paciente, visto que 0 oferecimento ao mesmo de fones de ouvido pode causar constrangimento e incômodo físico ou psicológico, principalmente dentre aqueles que perderam seus cabelos durante o tratamento (WANG X, et al., 2018).

Os resultados do estudo desenvolvido por Bieligmeyer $\mathrm{S}$, et al. (2018) que objetivou avaliar os efeitos imediatos de uma intervenção com música ambiente no que diz respeito ao bem-estar subjetivo, bem como 
ao calor corporal e à dor de pacientes mostraram fortemente que a musicoterapia aplicou resultados positivos no que diz respeito aos níveis de ansiedade, concepção sintomática, estresse e estado emocional. Porém, um ponto importante é a evidência de que a intervenção foi realizada a curto prazo e com pouca associação de parâmetros fisiológicos, sendo necessários mais estudos voltados para a prática de confirmação laboratorial.

Apesar dos mecanismos fisiológicos não terem sido completamente elucidados, os estudos utilizados apontaram a musicoterapia como apresentadora de um papel relevante sobre o sistema nervoso autônomo, causando a redução da liberação de catecolaminas refletindo em alterações da pressão arterial sistólica, pressão arterial diastólica, ocorrência de vômitos, além da liberação de neurotransmissores como dopamina e serotonina (GENCER D, et al., 2019; WANG X, et al., 2018.)

De modo geral, o câncer e seus tratamentos evidenciam uma série de modificações físicas, muito relacionadas a estética, que favorecem o desenvolvimento de sofrimento psicológico frente ao medo do futuro, sensação de fraqueza e despreparo para enfrentar a doença (GENCER D, et al., 2019). A dimensão tomada diante dessa sinestesia demonstra a importância do tema estudado, com o objetivo de retardar os processos que podem potencializar o estado doentio humano.

\section{CONSIDERAÇÕES FINAIS}

Diante dos artigos analisados na literatura científica, foi possível avaliar as características da musicoterapia em um panorama global, identificando diversos fatores relevantes para essa prática integrativa em pacientes com câncer em tratamento quimioterápico, tais como a redução de condições psicológicas (ansiedade, raiva, estresse, angústia e percepção da dor) e físicas (fadiga, dispneia, insônia, fraqueza muscular, náuseas). Esses achados sustentam as evidências de que a musicoterapia é efetiva como terapia alternativa e complementar, ficando evidente a necessidade de novos estudos que possam explorar determinantes laboratoriais com o intuito de ampliar os espectros fisiológicos avaliados nos pacientes submetidos à quimioterapia e os efeitos terapêuticos naqueles que recebem a intervenção musical.

\section{FINANCIAMENTO}

Este estudo foi financiado pelo Programa Institucional de Bolsas (PIBIC/CNPq) da Universidade José do Rosário Vellano - UNIFENAS, vigência 08/2019 a 07/2020.

\section{REFERÊNCIAS}

1. BARBOSA NERES C, et al. Efetividade da musicoterapia na redução da ansiedade de pacientes oncológicos: revisão sistemática. Rev Bras Cancerol, 2020; 65(4): e-08592.

2. BENENZON R. Teoria da musicoterapia: contribuição ao conhecimento do contexto não-verbal. 3ª ed. São Paulo: Summus, 1998; 184p.

3. BEYEA SC, NICOLL ELH. Writing an integrative review. Aorn J, 1998; 67(4): 877-880.

4. BIELIGMEYER S, et al. Feeling the sound - short-term effect of a vibroacoustic music intervention on well-being and subjectively assessed warmth distribution in cancer patients - a randomized controlled trial. Complement Ther Med, 2018; 40(1): 171-178.

5. BRADT J, et al. Music interventions for improving psychological and physical outcomes in cancer patients. Cochrane Database Syst Rev, 2016; 15(8): 1-157.

6. BRASIL. 2020. In: Instituto Nacional de Câncer José Alencar Gomes da Silva. Câncer de mama: Versão para Profissionais de Saúde. Disponível em: https://www.inca.gov.br/tipos-de-cancer/cancer-de-mama/profissional-desaude. Acesso em 12 out. 2020.

7. BRASIL. 2020. In: SUS de A a Z. Práticas integrativas e complementares. Disponível em: https://antigo.saude.gov.br/saude-de-a-z/praticas-integrativas-e-complementares. Acesso em 12 out. 2020.

8. BRO ML, et al. Effects of live music during chemotherapy in lymphoma patients: a randomized, controlled, multi-center trial. Supportive care in cancer, 2019; 27(10): 3887-3896.

9. BURNS D, et al. Differences between supportive music and imagery and music listening during outpatient chemotherapy and potential moderators of treatment effects. J Music Ther, 2018; 55(1): 83-108.

10. CHEN S, et al. Comparison of group vs self-directed music interventions to reduce chemotherapy-related distress and cognitive appraisal: an exploratory study. Support Care Cancer, 2018; 26(2): 461-469.

11. GALVÃO CM, et al. A prática baseada em evidências: considerações teóricas para sua implementação na enfermagem perioperatória. Rev Latino-am Enfermagem, 2002; 10(5): 690-695. 
12. GENCER D, et al. Cancer patients and music: (prospective) results from a survey to evaluate potential complementary treatment approaches. J Cancer Res Clin Oncol, 2019; 145(8): 2141-2148.

13. GREENLEE $\mathrm{H}$, et al. Clinical practice guidelines on the evidence-based use of integrative therapies during and following breast cancer treatment. CA Cancer J Clin, 2017; 67(3): 194-232.

14. GUYTON AC, HALL JE. Tratado de fisiologia médica. 13ª ed. Rio de Janeiro: Elsevier, 2017; 1176p.

15. LIMA TU, et al. Impact of a Music Intervention on Quality of Life in Breast Cancer Patients Undergoing Chemotherapy: A Randomized Clinical Trial. Integr Cancer Ther, 2020; 19: 1-9.

16. LIU H, et al. Effects of mindfulness-based stress reduction combined with music therapy on pain, anxiety, and sleep quality in patients with osteosarcoma. Braz J Psychiatry, 2019; 41(6): 540-545.

17. MASSIMILIANI V, et al. Music intervention during chemotherapy infusion reduces anxiety in oncological patients. European Society for Medical Oncology, 2017; 28(6): 82-88.

18. MELNYK BM, FINEOUT-OVERHOLT E. Making case for evidence-based practice. In: Melnyk BM; Fineout-Overholt E. Evidence-based practice in nursing \& healthcare. A guide to practice.4 ${ }^{\mathrm{a}}$ Philadelphia: Pippincot Williams \& Wilkins, 2018; 798p.

19. MENDES KDS, et al. Revisão integrativa: método de pesquisa para a incorporação de evidências na saúde e na enfermagem. Texto Contexto Enferm, 2008; 17(4): 758-764.

20. MERTZ BG, et al. Psychological distress among women with newly diagnosed breast cancer. Eur J Oncol Nurs, 2012; 16(4): 439-443.

21. PELLIZZON RF. Pesquisa na área da saúde: 1. Base de dados DeCS (Descritores em Ciências da Saúde). Acta Cir Bras, 2004; 19(2): 153-163.

22. RAMOS BF, LUSTOSA MA. Câncer de mama feminino e psicologia. Rev. SBPH, 2009; 12(1): 85-97.

23. ROBB S, et al. Pilot randomized trial of active music engagement intervention parent delivery for young children with cancer. J Pediatr Psychol, 2016; 42(2): 208-219.

24. SILVEIRA RCCP, GALVÃO CM. O cuidado de enfermagem e o cateter de Hickman: a busca de evidências científicas. Acta Paul. Enferm, 2005;18 (3): 276-284.

25. SOUZA MT, et al. Revisão integrativa: o que é e como fazer. Einstein, 2010; 8(1): 102-106.

26. TANG $\mathrm{H}-\mathrm{k}$, et al. Effect of musical therapy six steps on pain, anxiety and sleep quality in lung cancer patients undergoing chemotherapy. Basic And Clinical Pharmacology And Toxicology, 2015; 117(1): 1-17.

27. TOCCAFONDI A, et al. Live music intervention for cancer inpatients: The Music Givers format. Palliat Support Care, 2018; 16(6): 777-784.

28. URSI ES, GALVÃO CM. Prevenção de lesões de pele no perioperatório: revisão integrativa da literatura. Rev Latinoam Enfermagem, 2006; 14(1): 124-131.

29. URSI ES. Prevenção de lesões de pele no perioperatório: revisão integrativa da literatura. Dissertação (Mestrado em Ciências) - Escola de Enfermagem de Ribeirão Preto. Universidade de São Paulo, Ribeirão Preto, 2005; 130p.

30. WANG X, et al. Effects of music intervention on the physical and mental status of patients with breast cancer: a systematic review and meta-analysis. Breast Care, 2018; 13(3): 183-190. 